\title{
The Progress of Early Phase Bone Healing Using Porous Granules Produced from Calcium Phosphate Cement
}

\author{
P. Jungbluth ${ }^{1}$, M. Hakimi 1 , J. P. Grassmann' ${ }^{1}$ J. Schneppendahl ${ }^{1}$, A. Kessner ${ }^{1}$, M. Sager², A. R. Hakimi³, \\ J. Becker ${ }^{3}$, J. Windolf ${ }^{1}$, M. Wild ${ }^{1}$

\begin{abstract}
${ }^{1}$ Heinrich Heine University Hospital Duesseldorf, Department of Trauma and Handsurgery, Duesseldorf, Germany,
${ }^{2}$ Heinrich Heine University Hospital Duesseldorf, Animal Research Institute, Duesseldorf, Germany,

${ }^{3}$ Heinrich Heine University Hospital Duesseldorf, Department of Oral Surgery, Duesseldorf, Germany
\end{abstract}

\begin{abstract}
Objective: Bone grafting is a vital component in many surgical procedures to facilitate the repair of bone defects or fusions. Autologous bone has been the gold standard to date in spite of associated donor-site morbidity and the limited amount of available donor bone. The aim of this study was to investigate the progress of bone regeneration and material degradation of calcium phosphate granules (CPG) produced from a calcium phosphate self-setting cement powder compared to the use of autologous bone grafting in the treatment of "critical size defects" on load-bearing long bones of minipigs.
\end{abstract}

Methods: A critical size defect in the tibial metaphysis of 16 mini-pigs was filled either with autologous cancellous graft or with micro- and macroporous carbonated, apatic calcium phosphate granules (CPG) produced from a calcium phosphate self-setting cement powder. After 6 weeks, the specimens were assessed by $\mathrm{X}$-ray and histological evaluation. The amount of new bone formation was analysed histomorphometrically. Results: The semi-quantitative analysis of the radiological results showed a complete osseous bridging of the defect in three cases for the autograft group. In the same group five animals showed a beginning, but still incomplete bridging of the defect, whereas in the CPG group just two animals developed this. All other animals of the CPG group showed only a still discontinuous new bone formation. Altogether, radiologically a better osseous bridging was observed in the autograft group compared to the CPG group.

Histomorphometrical analysis after six weeks of healing revealed that the area of new bone was significantly greater in the autograft group concerning the central area of the defect zone $(\mathrm{p}<0.001)$ as well as the cortical defect zone $(p<0.002)$. All defects showed new bone formation, but only in the autograft group defects regenerated entirely

Conclusions: Within the limits of the present study it could be demonstrated that autologous cancellous grafts lead to a significantly better bone regeneration compared to the application of calcium phosphate granules (CPG) produced from a calcium phosphate self-setting cement powder after 6 weeks. In the early phase of bone-healing, the sole application of CPG appears to be inferior to the autologous cancellous grafts in an in vivo critical size defect on load-bearing long bones of mini-pigs.

Key words: Calcium phosphate granules; Calcium phosphate cement; Bone healing; Bone defect; Animal model; Mini-pig

\section{INTRODUCTION}

Bone grafting is a vital component in many surgical procedures to facilitate the repair of bone defects or fusions $[1,2]$. Autologous bone grafts are considered the gold standard in bone repair and regeneration, because of their osteogenicity, osteoinductivity and osteoconduction $[3,4]$. However, the harvesting procedure requires a second surgical site, at which complications have been reported [3]. Furthermore, the quantity of bone graft is limited $[5,6]$. For this reason a large number of artificial bone-substitute materials have been developed over the past few years in order to reduce the need to use an autograft or even go so far as to replace it $[7,8,9]$. The most frequent type are synthetic and biodegradable materials based on calciumphosphate $(\mathrm{CaP})$ such as $\beta$-tricalcium phosphates $(\beta$ TCP) or calcium-deficient hydroxyapatite (CDHA) in the form of ceramics or cements which, however, are primarily only of an osteoconductive nature [10]. At the same time, a distinction is made along the lines of the setting procedure between ceramics (sintering) and cements (sedimentation). After mixing in powder, the $\mathrm{CaP}$ cements can be applied directly as a paste which then sets in the defect at body temperature [11]. Various sintering steps are performed on ceramics at high temperatures in the production process, on the other hand, and ceramics generally are not degradable, or only decompose over very long periods of time [11]. In the search for biologically degradable substances, $\mathrm{CaP}$ cements have enjoyed growing popularity $[12,13$, $14,15]$. In addition to the biological degradation through osteoclastic resorption, these offer in addition a biocompatibility which is more bone-specific, thus constituting an alternative to the ceramic bone-substitute materials used to date $[12,14,15]$.

Ceramic implants cannot be shaped intraoperatively, which means that they can only be worked into different forms to a limited extent $[16,17]$. They moreover 
usually exhibit poorer mechanical properties [14, 17]. The shape of bone-substitute materials in addition has a sizeable impact on how they are worked. Thus, several experimental and clinical studies have shown that calcium-phosphate-based bone-substitute materials in the form of granules are particularly easy to work [1, 17, 18]. Granular calcium phosphate materials have well established biocompatibility and osteoconductivity [17]. However, nearly all of the granular calcium phosphate materials that have been evaluated so far are of ceramic nature, because in the past granules from CaP could only be obtained by sintering [15, 17]. Now using appropriate techniques granules can be made from $\mathrm{CaP}$ cements with very high surface areas that might be potent drug carriers or biologically very reactive $[14,15,16]$. In spite of the many positive features of $\mathrm{CaP}$ cements, there have only been a few in vivo studies in which these have been used in the form of granules to date $[12,14,16,19,20]$.

Consequently, an examination of the progress of bone regeneration and material degradation of microand macroporous $(50-550 \mu \mathrm{m}$ pores) carbonated, apatic calcium phosphate granules (CPG) (2 to $4 \mathrm{~mm}$ in size) produced from a calcium phosphate self-setting cement powder compared to the use of autologous bone grafting in the treatment of "critical size defects" on load-bearing long bones of mini-pigs was the subject of this study.

\section{Material AND Methods}

\section{Animals}

Sixteen female Goettinger mini-pigs (aged 18-30 months, weight $25-35 \mathrm{~kg}$ ) were used in this study. Animal selection, management and surgery protocol were approved by the Animal Care and Use Committee of the Heinrich Heine University and Bezirksregierung Duesseldorf (animal permit 50.05-230-78/06). The experimental segment of the study started after an adaptation period of two weeks.

\section{Study Design and Surgical Procedure}

The animals were randomized into the CPG and the autograft group. Each group consisted of 8 mini-pigs. According to Wheeler et al. [21] in all animals an 11 $\mathrm{mm}$ cylindrical defect from medial to lateral to a depth of $25 \mathrm{~mm}$ at the right proximal tibia without lateral cortical penetration was surgically created using a cannulated reamer (Aesculap AG \& Co. KG, Tuttlingen, Germany). In one group the defect was filled with micro- and macro-porous (50-550 $\mu \mathrm{m}$ pores) carbonated, apatic calcium phosphate granules (CPG) (2 to $4 \mathrm{~mm}$ in size) produced from a calcium phosphate self-setting cement powder (Calcibon ${ }^{\circledR}$ Granules, Biomet Deutschland $\mathrm{GmbH}$, Berlin, Germany). In the other group the defect was filled with an autologous cancellous graft. In all cases the animals were permitted to walk under full weight bearing immediately after surgery. None of them suffered from a tibial fracture or had to be sacrificed ahead of schedule.

All animals were starved for a minimum of $12 \mathrm{~h}$ before surgery. Antibiotic preparation was given once to each animal in a perioperative way as single shot of 3.3 ml Lincomycin (Lincomycin 20\%, WDT, Garbsen, Germany). After intramuscular sedation with 0.5 $\mathrm{mg} / \mathrm{kg}$ Atropin (Atropinsulfat, B Braun, Melsungen, Germany), $5 \mathrm{mg} / \mathrm{kg}$ Azaperon (/Stresnil ${ }^{\circledR}$, JanssenCilag GmbH, Neuss, Germany) and $10 \mathrm{mg} / \mathrm{kg}$ Ketamin $\left(\right.$ Ketavet $^{\circledR}$, Pharmacia GmbH, Karlsruhe, Germany), anaesthesia was initiated using $0.5 \mathrm{~g}$ Thiopental (Inresa Arzneimittel GmbH, Freiburg, Germany). For all surgical procedures, inhalation anaesthesia was performed by use of oxygen and nitrous oxide and isoflurane. To maintain hydration and cardiac protection, all animals received a constant rate infusion of $5 \%$ Glucose's solution (Delta-Select, Pfullingen, Germany) combined with $10 \mathrm{ml}$ Inzolen (Koehler Chemie $\mathrm{GmbH}$, Alsbach-Hähnlein, Germany) and $5 \mathrm{ml}$ Lidocain 2\% (Lidocain- $\mathrm{HCl}, \mathrm{B}$. Braun, Melsungen, Germany) while anaesthetized. Intraoperative analgesia was performed by intravenous injection of $0.4 \mathrm{mg} / \mathrm{kg}$ Piritramid (Dipidolor ${ }^{\circledR}$, Janssen-Cilag GmbH, Neuss, Germany) and $4.5 \mathrm{mg} / \mathrm{kg}$ Carprofene (Rimadyl ${ }^{\circledR}$, Pfitzer Pharma GmbH, Karlsruhe, Germany). For postoperative treatment, piritramid and carprofene were applied subcutaneously for 3 days at the same dose. Additionally, prophylactic administration of Lincomycin (3.3 ml Lincomycin 20\%, WDT, Garbsen, Germany) was performed postoperatively for 3 days.

All surgical procedures took place under aseptic conditions and were performed by the same experienced surgeon. In the autograft group the left iliac crest was shaved, disinfected by Cutasept ${ }^{\circledR}$ (Bode, Hamburg, Germany) and sterilely draped. An incision was made over the left iliac crest and sharp dissection was used to expose the bone. A Kirschner wire (Kwire) was inserted in the iliac crest. The cancellous graft was harvested from the iliac crest using the same cannulated reamer of $11-\mathrm{mm}$ caliber. The donor site was packed with gauze sponges until bleeding was controlled and the incision closed in layers.

In both groups the right limb, distal to mid-thigh was shaved, desinfected by Cutasept ${ }^{\circledR}$ (Bode, Hamburg, Germany) and sterilely draped. The right proximal tibia was exposed using a medial approach and soft tissue was reflected. The joint surface and the anterior and most posterior extent of the tibial plateau were identified. A Kirschner-wire (K-wire) was inserted 10 $\mathrm{mm}$ distal to the joint line and $12 \mathrm{~mm}$ anterior to the most posterior aspect of the tibia. An $11 \mathrm{~mm}$ cylindrical defect was created using the aforementioned cannulated reamer, drilling from medial to lateral to a depth of $25 \mathrm{~mm}$ without lateral cortical penetration. The defect was rinsed copiously with saline and packed with gauze to control bleeding. The defect was filled with the appropriate graft material and the graft gently compressed with a bone tamp. In the autograft group the size of the harvested graft was almost identical to the proximal tibial defect we created. In all cases the amount of cancellous graft was adequate to fill the defect. In the CPG group the amount of CPG reflected the size of the defect and was adequate to fill the defect in all cases. Soft tissues were closed in layers.

In preliminary experiments the same defect was created in the proximal tibia of four minipigs without any additional filling material. All four animals had to be sacrificed prematurely due to a proximal tibia frac- 
ture between the first and third postoperative day. For this reason the defect model in question fulfils the criteria of a critical size defect. In order to avoid the loss of further animals and due to ethical reasons this study does without a control group lacking grafting material.

\section{FOLLOW UP AND SACRIFICES}

Postoperatively, the animals were housed in individual pens. In all cases the animals were permitted to walk immediately after surgery. Body temperature, body weight and general conditions were examined until completed wound healing. After 6 weeks animals were sacrificed by overdose of sodium pentobarbital 3\% (Eutha 77, Essex Pharma GmbH, München, Germany). The proximal tibia was dissected from the distal femoral shaft to the proximal tibial shaft and the soft tissues removed. All specimens were fixed in 10\% neutral buffered formalin solution for 14 days.

\section{RADiological Evaluation}

Conventional X-rays in two planes were obtained on the explanted tibial bones after 6 weeks (Heliodent DS, Sirona Dental Systems GmbH, Bensheim, Germany). All X-rays were evaluated by a sole experienced radiologist masked to the specific experimental conditions. According to Sarkar et al. [22] the degree of new bone formation was estimated using a semi-quantitative score from 0 (no mineralized bone) up to at best 4 (complete bridging of the defect with mineralized bone). The other score values were defined as follows: few and isolated centers of ossification (1); more, but still discontinuous new bone formation (2); beginning, but incomplete bridging of the defect (3).

\section{Histological Preparation}

The specimens were dehydrated using ascending grades of alcohol and xylene, infiltrated, and embedded in methylmethacrylate (Technovit 9100 NEU, Heraeus Kulzer, Wehrheim, Germany) for non-decalcified sectioning. During this procedure, any negative influence of polymerisation heat was avoided by performing controlled polymerization in a cold atmosphere $\left(-4{ }^{\circ} \mathrm{C}\right)$. After $20 \mathrm{~h}$ the specimens were completely polymerized. Each specimen was cut in the axial direction using a diamond wire saw $\left(\right.$ Exakt $^{\circledR}$, Apparatebau, Norderstedt, Germany). Serial sections were prepared from the central parts of the defect areas, resulting in three sections each of approximately $320 \mu \mathrm{m}$ thickness [23,24]. All specimens were glued with acrylic cement (Technovit 7210 VLC, Heraeus Kulzer) to oversize plastic slides (size: $50 \times 100 \times 2 \mathrm{~mm}$, DiaPlus, Walter Messner GmbH, Oststeinbeck, Germany) and ground to a final thickness of approximately 60 $\mu \mathrm{m}$. All the sections were scheduled for histomorphometric analysis and stained with toluidine blue.

\section{HistomORPHOMETRICAL ANALYSIS}

Histomorphometrical analyses as well as microscopic observations were performed by one experienced in- vestigator masked to the specific experimental conditions. For image acquisition a colour CCD camera (Colour View III, Olympus, Hamburg, Germany) was mounted on a binocular light microscope (Olympus SZ 61, Olympus). Digital images (original magnification X 6.7) were evaluated using a software program (Cell D, Olympus GmbH, Hamburg, Germany). The quantitative analysis of new bone formation (area of new-bone formation in $\mu \mathrm{m}^{2}$ and percentage of total new-bone formation) was measured histomorphometrically in two predefined regions of interest. One was positioned in the cortical defect zone and the other one in the central area of the defect zone (Fig. 1.). Three sections were evaluated for each bone defect. Additionally, the amount of osseous bridging in the cortical defect zone as well as the osseous bridging in the central area of the defect zone were evaluated under standard light microscopy (original magnification $\mathrm{x} 20$ ) using a semiquantitative score from 0 (no bridging), 1 (incomplete bridging) up to at best 2 (complete bridging of the defect with mineralized bone).

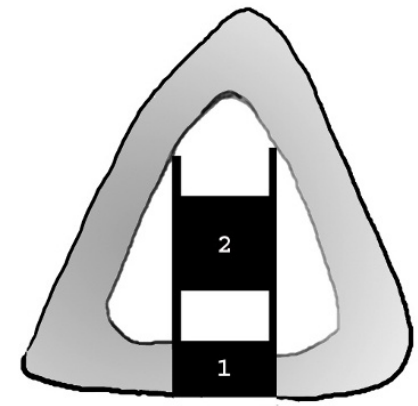

Fig. 1. Scheme of a histological slide with the bone defect in the proximal tibia. The quantitative analysis of new bone formation was measured histomorphometrically in two regions of interest. 1. the cortical defect zone (size: 1500 x 7000 pixels) 2. the central defect zone (size: $3000 \times 7000$ pixels)

\section{StATISTICAL ANALYSIS}

The statistical analysis was performed using a commercially available software program (SPSS 17.0, SPSS Inc., Chicago, IL, USA). Mean values and standard deviations were calculated for each group. For the statistical comparisons between groups, the unpaired t-test was used. Significance was defined as a $\mathrm{p}$ value $<0.05$.

\section{RESULTS}

The postoperative healing was uneventful in all pigs. No complications such as allergic reactions, abscesses or infections were observed throughout the study period.

\section{RADiOLOGical Evaluation}

Evaluating the $\mathrm{X}$-rays areas of mineralization were visible after 6 weeks in all defects of the autograft group as well as the CPG group. Complete osseous bridging (score $=4)$ was observed in three mini-pigs of the autograft group. In the same group five animals showed 
a beginning, but still incomplete bridging of the defect, whereas in the CPG group just two animals developed this (score $=3$ ). All other animals of the CPG group showed only a still discontinuous new bone formation $($ score $=2$ ) (Fig. 2.). Altogether, radiologically
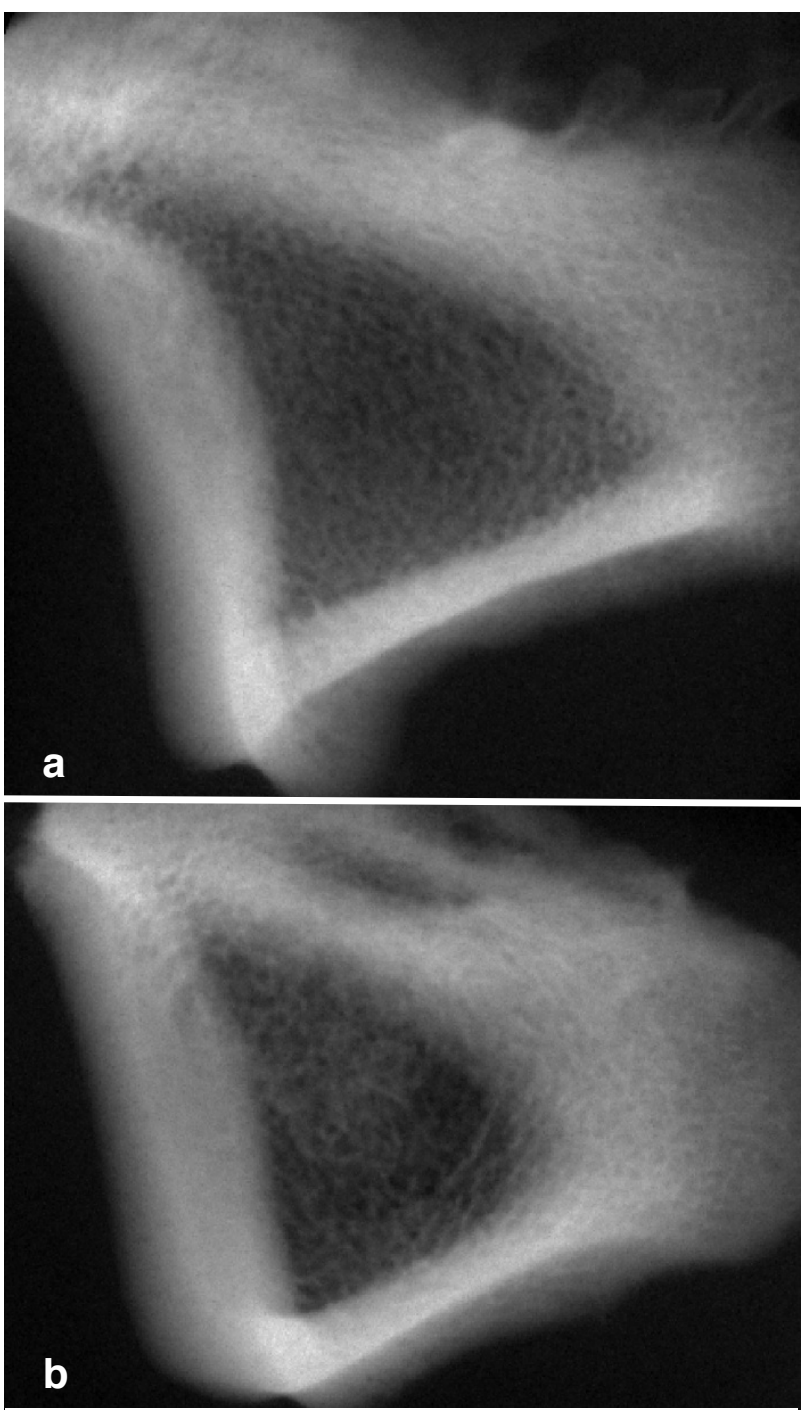

Fig. 2. Semiquantitative radiological evaluation. a) X-rays of the defect area showing a complete osseous bridging (score $=4$ ), applying autograft from the iliac crest. b) X-rays of the defect area showing a still discontinuous new bone formation (score $=2$ ), applying calcium phosphate granules. a better osseous bridging was observed in the autograft group compared to the CPG group.

\section{HistologicAl Results}

Histomorphometrical analysis after six weeks of healing revealed that the area of new bone was significantly greater in the autograft group concerning the central area of the defect zone $(p<0.001)$ as well as the cortical defect zone $(p<0.002)$ (Fig. 3.). All defects showed new bone formation, but only in the autograft group defects regenerated entirely (Fig. 4.). Occasionally, in the CPG group calcium phosphate granules were embedded in newly formed bone. Evidently, in these cases the newly formed bone was guided over the surfaces of the granules. Overall, only in small isolated areas of the defects within the granules newly formed bone infiltrated its' pores (Fig. 5.). In general, the histological findings were in accordance with the results of the histomorphometrical analysis and the $\mathrm{X}$-rays. There was regular bone healing in all animals of both groups. The sections stained with toluidine blue showed a physiological bone remodelling in all specimens. The presence of multinucleated giant cells/macrophages was more prominent in the autograft group. At the margins of the central area of the defect zone both resorption and new bone formation could be observed more intensively in the autograft group and stronger remodelling was seen in the adjoining cortical bone. The autograft group was superior to the CPG group even in the semiquantitative assessment of the osseous bridging in both observed areas of the defect (Fig. 6.). No inflammatory reaction was found in relation to any of the grafting materials.

\section{Discussion}

The findings of this study indicate that the results of the bone regeneration of metaphyseal defects on minipigs after 6 weeks with only CPG being used were inferior to the results produced by autologous cancellous grafting. In this context, the semi-quantitative analysis of the radiological results of the metaphyseal defects to the proximal tibia of the mini-pigs after six weeks showed a complete osseous bridging of the defect in three cases for the autograft group. In the same group five animals showed a beginning, but still incomplete bridging of the defect. In the CPG group, however, just two animals developed a beginning, but

\begin{tabular}{|c|c|c|c|}
\hline Method & & $\begin{array}{c}\text { Cortical defect zone } \\
\text { Area of newly formed bone / total } \\
\text { defect area [\%] }\end{array}$ & $\begin{array}{c}\text { Central defect zone } \\
\text { Area of newly formed bone / total } \\
\text { defect area [\%] }\end{array}$ \\
\hline Autograft & $\begin{array}{c}\text { mean } \\
\text { standard deviation }\end{array}$ & 39.3 & 37.5 \\
CPG & mean & 7.4 & 7.9 \\
& standard deviation & 24.7 & 9.8 \\
\end{tabular}

Fig. 3. Histomorphometrical analysis of the area of new bone in relation to the total defect area concerning the central as well as the cortical defect zone. In both defect zones the area of new bone was significantly higher in the autograft group (central area of the defect zone: $p<0.001$ autograft group versus CPG group, cortical defect zone: $p<0.002$ autograft group versus CPG group). 

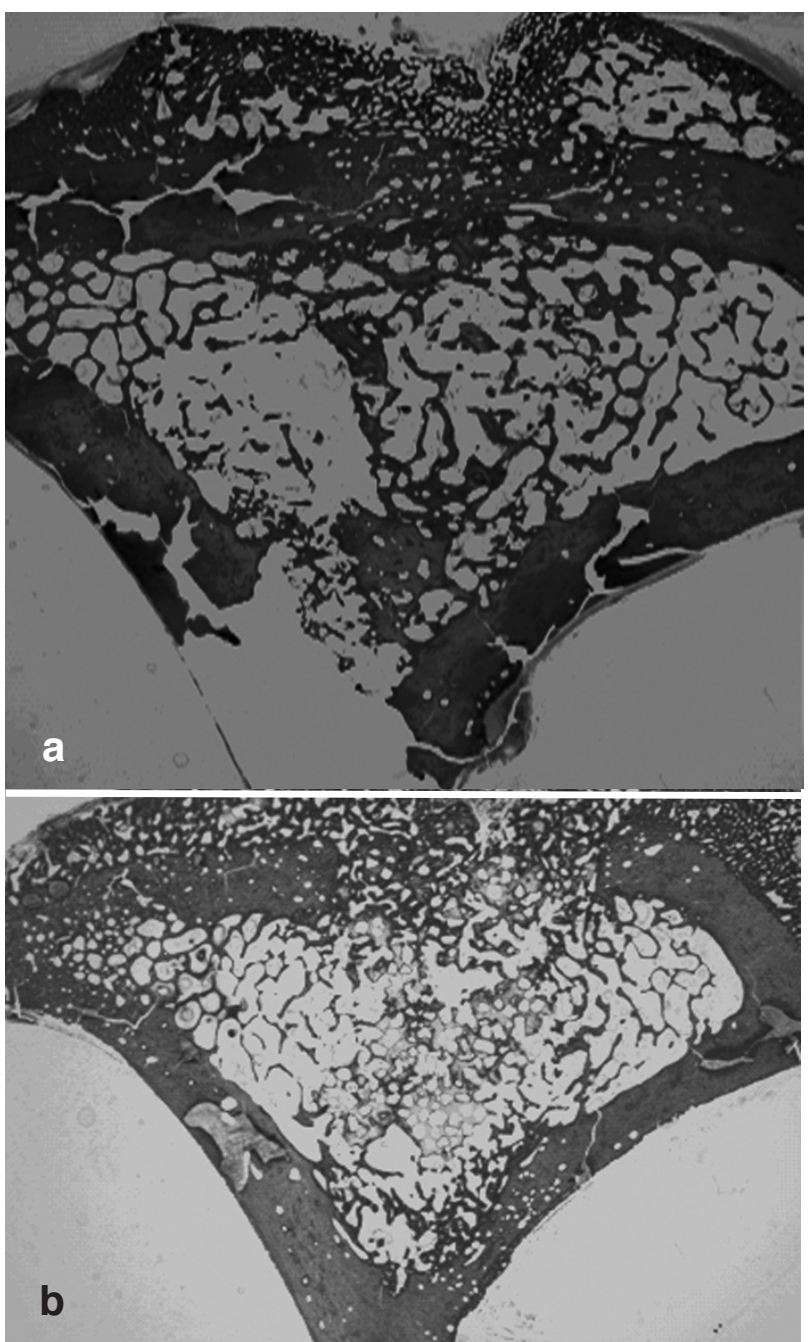

Fig. 4. Histological sections of the autograft and CPG group 6 weeks after surgery (original magnification X 6.7). Four defects of the autograft group (a) regenerated entirely. a: Autograft group, b: CPG group.

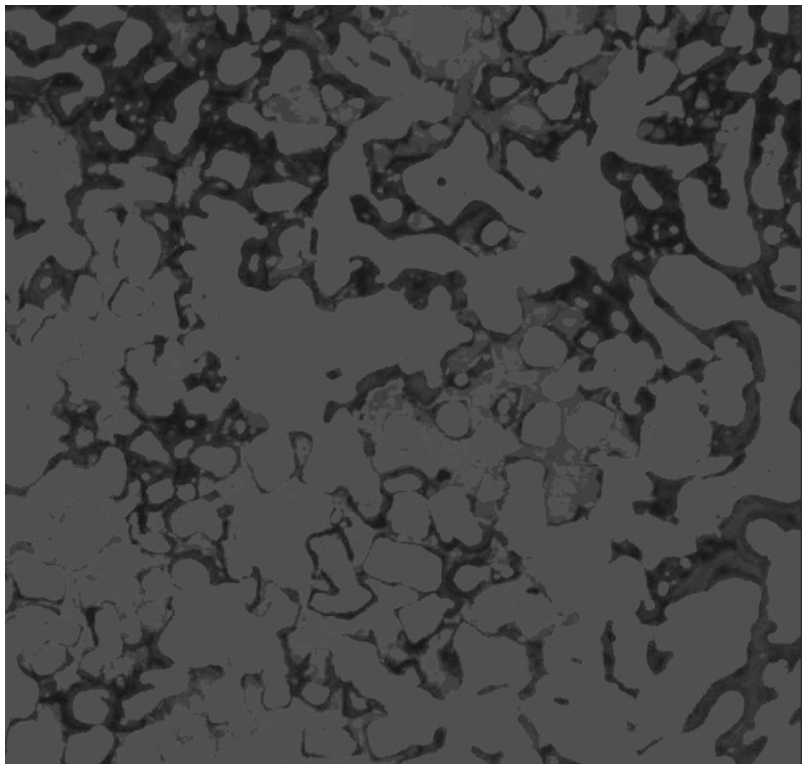

Fig. 5. Histological sections of the CPG group 6 weeks after surgery (original magnification X 20). Only some of the calcium phosphate granules are embedded in newly formed bone.
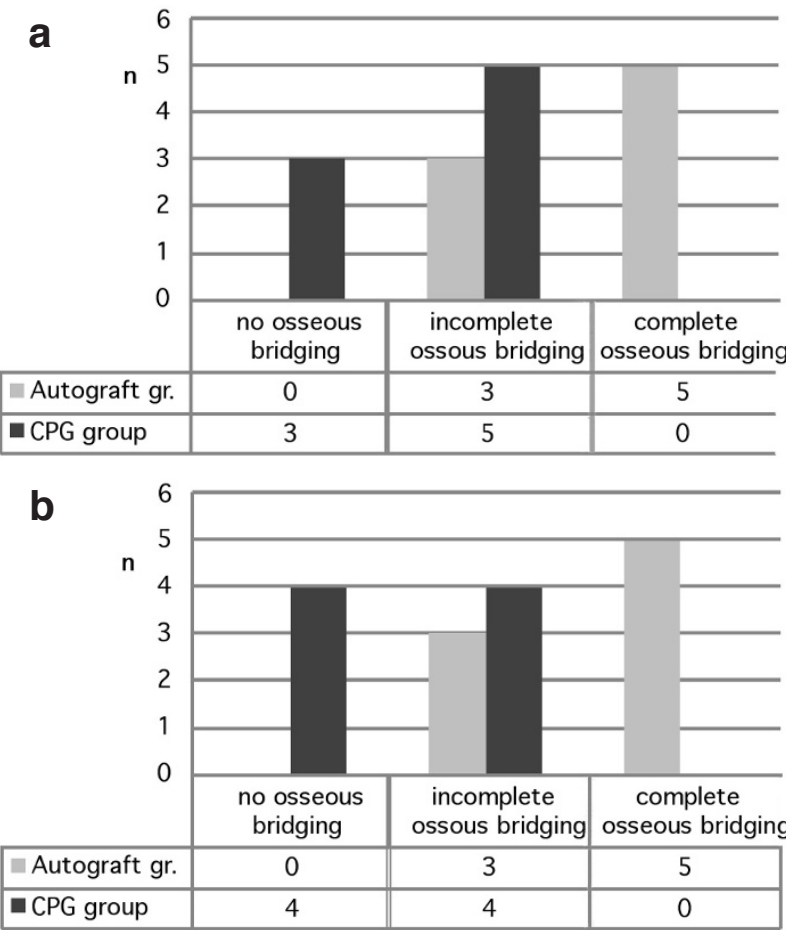

Fig. 6. Semiquantitative analysis of osseous bridging. a: Semiquantitative analysis of the cortical defect zone, b: Semiquantitative analysis of the central defect zone.

These findings were supported by the histomorphometrical data. Here, a significantly superior formation of new bone was found both in the central and in the cortical defect zone in the autograft group. Furthermore, the autograft group was superior to the CPG group even in the semiquantitative assessment of the osseous bridging in both observed areas of the defect.

$\mathrm{CaP}$ ceramics have been used successfully for metaphyseal bone defects in many instances as a possible alternative to cancellous grafting $[3,21,25,26,27]$. In comparison to $\mathrm{CaP}$ ceramics, on the other hand, $\mathrm{CaP}$ cements possess several advantages such as, for example, a larger specific surface area, better biological degradability through osteoclastic resorption and a more specific biocompatibility [14, 15]. Nevertheless, within the framework of our study, we were not able to establish results which were as good as those produced by $\mathrm{CaP}$ ceramics with calcium phosphate granules (CPG) produced from a calcium phosphate selfsetting cement powder in the early phase of defect healing in the filling of bone defects on load-bearing long bones, although the literature results relating to $\mathrm{CaP}$ ceramics are based on long-term observations.

Wheeler et al. were able to demonstrate a significantly higher formation rate of new bone in an animal study on goats using $\beta$-TCP ceramics in particle form in a metaphyseal critical-size defect on the proximal tibia, which is very similar to our defect model, in comparison to the autograft group. However, these followup examinations were only performed 3 months later here [21]. Using a histomorphometrical assessment comparable with the method we used, the authors demonstrated a bone regeneration of $24 \%$ in the central defect zone, while approximately $15 \%$ of bone re- 
generated in the same region in the autograft group [21]. In comparison to this, we were able to demonstrate in our study that there was a significantly greater bone formation in comparison to the CPG group both in the central $(37.5 \%: 9.8 \%)$ as well as in the cortical defect zone (39.3\%:24.7\%) after 6 weeks in the autograft group. In contrast to Wheeler et al., mini-pigs were deliberately used as the laboratory animal in our study with a comparable defect model, as their new bone formation rate of $1.2-1.5 \mathrm{~mm} /$ day is almost identical to the reparative osseous capacities of humans [28].

In animal studies, Lange et al. arrived at similar results as Wheeler et al. after filling in a metaphyseal tibial defect on a mini-pig with TCP ceramic particles [25]. These authors demonstrated 13\% newly formed bone in the central defect zone in the autograft group and $32 \%$ newly formed bone in the TCP group [25]. This evaluation was only performed 9 months later, however, and thus like in the case of Wheeler et al. much later than in our study.

The primary cement-powder, and hence the input material for the $\mathrm{CaP}$ granules under examination here, has already been successfully used in animal studies as well as in clinical studies, and have been described as biocompatible and osteoconductive and an alternative to autograft $[18,29]$. Very little data has been published to date on the use of this cement in the form of granules for critical-size defects [19, 20, 29]. According to Bohner and Baumgart, the in vivo resorption rate of porous granules can be predicted to be much faster than in dense blocks or prismatic objects made out of the same material [30]. It should be noted, however, that direct comparisons of TCP-ceramics in granular form have not been well reported, either [1]. Walsh et al. evaluated bone formation and implant resorption of three TCP-ceramic bone graft substitutes of similar chemistry but different porosity in granular form in a standardized tibial defect model in rabbits [1]. The authors were able to demonstrate, that all three scaffolds were osteoconductive and supported new bone formation while implant resorption with time differed between materials [1]. The scaffold with the greatest porosity $(90 \%)$ and the largest pore size $(1-1000 \mu \mathrm{m})$ resorbed faster than the other materials (porosities of $75 \%$, pore sizes of $100-400 \mu \mathrm{m}$ ) [1]. Hence, implant porosity and pore structure seem to play an important role in the in vivo resorption and new bone ingrowth $[1,14,16]$. This is also confirmed by other authors, interconnections or pathways between the pores are also vital for new bone integration into the material $[31,32,33]$. In comparison to the TCP ceramics used by Walsh et al., the CaP granules used in our study exhibited both a lower porosity $(50 \%)$ and pore sizes $(50-550 \mu \mathrm{m})$. This could serve as an explanation for the lower new bone-formation rate. This was also the impression gained in our histological examinations. Here, occasionally, in the CPG group $\mathrm{CaP}$ granules were embedded in newly formed bone. Evidently, in these cases the newly formed bone was guided over the surfaces of the granules. Overall, only in small isolated areas of the defects within the granules newly formed bone infiltrated its' pores. It was probably the case that the lower porosity and pore size prevented a greater migration of cells such as e.g. os- teoclasts and osteoblasts into the granules and thus a further bony in-growth within the three-dimensional geometry of the scaffold. The reason for this is that in comparison to sintered $\mathrm{CaP}$ materials, two unique features are actually attributed to $\mathrm{CaP}$ cement granules in particular [14]. Firstly, apatite CPCs are nanocrystalline and hence have a very high specific surface area [14]. Values as high as $100 \mathrm{~m}^{2} / \mathrm{g}$ can be reached [14]. By comparison, sintered ceramics have surface areas close to or below $1 \mathrm{~m}^{2} / \mathrm{g}[14,17]$. Secondly, CPC-granules consist of low temperature calcium phosphates such as dicalcium phosphate dehydrate or precipitated apatite. These hydrated compounds can all be found in the body, contrary to traditional bone substitute ceramics such as sintered hydroxylapatite, $\beta$-tricalcium phosphate and biphasic calcium phosphates [14]. There have, however, so far only been a limited number of clinical and experimental studies conducted to test the feasibility of these $\mathrm{CaP}$ cement-granules, which are moreover difficult to compare with our animal model. In vitro cell culture tests $[34,35,36]$ and only few in vivo implantations have been performed $[19,20]$. However, there is presently no in vivo study clearly demonstrating differences in performance between $\mathrm{CaP}$ cement-granules and sintered ceramic granules [14]. There is also no study linking the surface properties of CaP-cement-granules and their in vivo performance [14]. CaP cement granules could offer a crucial advantage here in particular: due to their high specific surface area. CaP cement -granules have a large potential as drug carriers or drug delivery system [14]. New trends in this connection are the increasing use of bone-substitute materials in combination with innovative substances such as, for example, platelet-rich plasma (PRP) and/or mesenchymal stem cells $[6,22,37,38,39$, 40, 41]. Kasten et al. demonstrated that bone substitutes with high specific surface areas cause more extensive and longer absorption of the PRP which leads to the infiltration of new bone deep within and not just on the outer surface of the materials [6, 39, 40]. Moreover, Tas et al. demonstrated that the same calcium phosphate granules as we used in our study are able to almost fully absorb citrated blood or fluid PRP prior to their implantation, which is based on a high wicking ability of the granules [12].

Even if within the limitations of this study the bony healing results produced by the exclusive use of CPG in the case of metaphyseal defects on mini-pigs were inferior to autologous cancellous grafting, this bone-substitute material holds out considerable potential as a satisfactory drug delivery system as a result of its high specific surface area. The additional use of innovative substances such as, for example, platelet-rich plasma (PRP) and/or autologous mesenchymal stem cells (MSC) should in this context serve as the starting point for additional studies.

\section{REFERENCES}

1. Walsh Wr, Vizesi F, Michael D, Auld J, Langdown A, Oliver R, Yu Y, Irie HandBruce W. Beta-TCP bone graft substitutes in a bilateral rabbit tibial defect model. Biomaterials. 2008;29: 266-71. 
2. Perry Cr. Bone repair techniques, bone graft, and bone graft substitutes. Clin Orthop Relat Res. 1999: 71-86.

3. Fellah Bh, Gauthier O, Weiss P, Chappard DandLayrolle P. Osteogenicity of biphasic calcium phosphate ceramics and bone autograft in a goat model. Biomaterials. 2008; 29: $1177-88$

4. Cedidi Cc, Felmerer GandBerger A. Management of defects in the groin, thigh, and pelvic region with modified contralateral TRAM/VRAM flaps. Eur J Med Res. 2005; 10: $515-20$

5. Arrington Ed, Smith Wj, Chambers Hg, Bucknell AlandDavino Na. Complications of iliac crest bone graft harvesting. Clin Orthop Relat Res. 1996: 300-9.

6. Kasten P, Vogel J, Geiger F, Niemeyer P, Luginbuhl RandSzalay K. The effect of platelet-rich plasma on healing in critical-size long-bone defects. Biomaterials. 2008;29: 3983-92.

7. Cornell $\mathrm{Cn}$. Osteoconductive materials and their role as substitutes for autogenous bone grafts. Orthop Clin North Am. 1999;30:591-98.

8. Petite H, Viateau V, Bensaid W, Meunier A, De Pollak C, Bourguignon M, Oudina K, Sedel LandGuillemin G. Tissue-engineered bone regeneration. Nat Biotechnol. 2000;18: 959-63.

9. Okafuji N, Shimizu T, Watanabe T, Kimura A, Kurihara S, Arai Y, Furusawa K, Hasegawa HandKawakami T. Three-dimensional observation of reconstruction course of rabbit experimental mandibular defect with rhBMP-2 and atelocollagen gel. Eur J Med Res. 2006;11: 35154.

10. Legeros Rz. Properties of osteoconductive biomaterials: calcium phosphates. Clin Orthop Relat Res. 2002: 81-98.

11. Schieker M, Heiss CandMutschler W. [Bone substitutes]. Unfallchirurg. 2008;111: 613-19; quiz 620.

12. Tas Ac. Preparation of porous apatite granules from calcium phosphate cement. J Mater Sci Mater Med. 2008;19 :2231-39.

13. Burger ElandPatel V. Calcium phosphates as bone graft extenders. Orthopedics. 2007;30: 939-42.

14. Bohner M, Gbureck UandBarralet Je. Technological issues for the development of more efficient calcium phosphate bone cements: a critical assessment. Biomaterials. 2005;26: 6423-29.

15. Bohner M. Calcium orthophosphates in medicine: from ceramics to calcium phosphate cements. Injury. 2000;31 Suppl 4: 37-47.

16. Bohner M, Van Lenthe Gh, Grunenfelder S, Hirsiger W, Evison RandMuller R. Synthesis and characterization of porous beta-tricalcium phosphate blocks. Biomaterials. 2005;26: 6099-105.

17. Chow Lc. Next generation calcium phosphate-based biomaterials. Dent Mater J. 2009;28: 1-10.

18. Hillmeier J, Grafe I, Da Fonseca K, Meeder Pj, Noldge G, Libicher M, Kock Hi, Haag MandKasperk C. [The evaluation of balloonkyphoplasty for osteoporotic vertebral fractures. An interdisciplinary concept]. Orthopade. 2004;33: 893-904.

19. Kasten P, Luginbuhl R, Vogel J, Niemeyer P, Weiss S, Van Griensven M, Krettek C, Bohner M, Bosch UandTonak M. [Induction of bone tissue on different matrices: an in vitro and a in vivo pilot study in the SCID mouse]. Z Orthop Ihre Grenzgeb. 2004;142: 467-75.

20. Steffen T, Stoll T, Arvinte TandSchenk Rk. Porous tricalcium phosphate and transforming growth factor used for anterior spine surgery. Eur Spine J. 2001;10 Suppl 2: S132-40.

21. Wheeler Dl, Cross Ar, Eschbach Ej, Rose At, Gallogly Pm, Lewis DdandVander Griend Ra. Grafting of massive tibial subchondral bone defects in a caprine model using beta-tricalcium phosphate versus autograft. J Orthop Trauma. 2005;19: 85-91.
22. Sarkar Mr, Augat P, Shefelbine Si, Schorlemmer S, Huber-Lang M, Claes L, Kinzl LandIgnatius A. Bone formation in a long bone defect model using a platelet-rich plasma-loaded collagen scaffold. Biomaterials. 2006;27: 1817-23.

23. Donath K. The diagnostic value of the new method for the study of undecalcified bones and teeth with attached soft tissue (Sage-Schliff (sawing and grinding) technique). Pathol Res Pract. 1985;179: 631-33.

24. Schwarz F, Herten M, Ferrari D, Wieland M, Schmitz L, Engelhardt EandBecker J. Guided bone regeneration at dehiscence-type defects using biphasic hydroxyapatite + beta tricalcium phosphate (Bone Ceramic) or a collagencoated natural bone mineral (BioOss Collagen): an immunohistochemical study in dogs. Int J Oral Maxillofac Surg. 2007;36: 1198-1206.

25. Lange Ta, Zerwekh Je, Peek Rd, Mooney VandHarrison Bh. Granular tricalcium phosphate in large cancellous defects. Ann Clin Lab Sci. 1986;16: 467-72.

26. Jensen Ss, Bornstein Mm, Dard M, Bosshardt DdandBuser D. Comparative study of biphasic calcium phosphates with different HA/TCP ratios in mandibular bone defects. A long-term histomorphometric study in minipigs. J Biomed Mater Res B Appl Biomater. 2009;90: 17181.

27. Wiltfang J, Merten Ha, Schlegel Ka, Schultze-Mosgau S, Kloss Fr, Rupprecht SandKessler P. Degradation characteristics of alpha and beta tri-calcium-phosphate (TCP) in minipigs. J Biomed Mater Res. 2002;63: 115-21.

28. Schlegel Ka, Kloss Fr, Schultze-Mosgau S, Neukam FwandWiltfang J. [Osseous defect regeneration using autogenous bone alone or combined with Biogran or Algipore with and without added thrombocytes. A microradiologic evaluation]. Mund Kiefer Gesichtschir. 2003;7: 112-18.

29. Ooms Em, Wolke Jg, Van De Heuvel Mt, Jeschke BandJansen Ja. Histological evaluation of the bone response to calcium phosphate cement implanted in cortical bone. Biomaterials. 2003;24: 989-1000.

30. Bohner MandBaumgart F. Theoretical model to determine the effects of geometrical factors on the resorption of calcium phosphate bone substitutes. Biomaterials. 2004;25: 3569-82.

31. Lu Jx, Flautre B, Anselme K, Hardouin P, Gallur A, Descamps MandThierry B. Role of interconnections in porous bioceramics on bone recolonization in vitro and in vivo. J Mater Sci Mater Med. 1999;10: 111-20.

32. Habibovic P, Yuan H, Van Der Valk Cm, Meijer G, Van Blitterswijk CaandDe Groot K. 3D microenvironment as essential element for osteoinduction by biomaterials. Biomaterials. 2005;26: 3565-75.

33. Hing Ka, Annaz B, Saeed S, Revell PaandBuckland T. Microporosity enhances bioactivity of synthetic bone graft substitutes. J Mater Sci Mater Med. 2005;16: 46775.

34. Reinstorf A, Ruhnow M, Gelinsky M, Pompe W, Hempel U, Wenzel KwandSimon P. Phosphoserine--a convenient compound for modification of calcium phosphate bone cement collagen composites. J Mater Sci Mater Med. 2004;15: 451-55.

35. Kasten P, Luginbuhl R, Van Griensven M, Barkhausen T, Krettek C, Bohner MandBosch U. Comparison of human bone marrow stromal cells seeded on calcium-deficient hydroxyapatite, beta-tricalcium phosphate and demineralized bone matrix. Biomaterials. 2003;24: 2593603.

36. Hempel U, Reinstorf A, Poppe M, Fischer U, Gelinsky M, Pompe WandWenzel Kw. Proliferation and differentiation of osteoblasts on Biocement D modified with collagen type I and citric acid. J Biomed Mater Res B Appl Biomater. 2004;71: 130-43. 
37. Plachokova As, Van Den Dolder J, Stoelinga PjandJansen Ja. Early effect of platelet-rich plasma on bone healing in combination with an osteoconductive material in rat cranial defects. Clin Oral Implants Res. 2007;18: 244-51.

38. Nair Mb, Varma Hk, Menon Kv, Shenoy SjandJohn A. Reconstruction of goat femur segmental defects using triphasic ceramic-coated hydroxyapatite in combination with autologous cells and platelet-rich plasma. Acta Biomater. 2009;5: 1742-55.

39. Kasten P, Vogel J, Luginbuhl R, Niemeyer P, Weiss S, Schneider S, Kramer M, Leo AandRichter W. Influence of platelet-rich plasma on osteogenic differentiation of mesenchymal stem cells and ectopic bone formation in calcium phosphate ceramics. Cells Tissues Organs. 2006; 183: 68-79.

40. Kasten P, Vogel J, Beyen I, Weiss S, Niemeyer P, Leo AandLuginbuhl R. Effect of platelet-rich plasma on the in vitro proliferation and osteogenic differentiation of human mesenchymal stem cells on distinct calcium phosphate scaffolds: the specific surface area makes a difference. J Biomater Appl. 2008;23: 169-88.
41. Kilian O, Flesch I, Wenisch S, Taborski B, Jork A, Schnettler RandJonuleit T. Effects of platelet growth factors on human mesenchymal stem cells and human endothelial cells in vitro. Eur J Med Res. 2004;9: 337-44.

Received: February 24,2010 / Accepted: March 11, 2010

Address for correspondence:

Mohssen Hakimi, M.D.

Heinrich Heine University Hospital Duesseldorf

Department of Trauma and Handsurgery

Moorenstr. 5

40225 Duesseldorf, Germany

Tel.: $\quad+49211 / 8104404$

Fax: $\quad+49211 / 8104902$

E-mail: mohssen.hakimi@med.uni-duesseldorf.de 